hemorrhage in the second and third decades of life.

The inclusion of milder sickle syndromes, and subtle neurologic abnormalities related to a low hematocrit, emphasizes the need for closer patient follow-up and studies of the risk/benefit ratio of chronic prophylactic transfusion therapy or alternative treatments (Adams RJ et al. N Engl I Med 1998;339:5-11).

\title{
VERY BRIEF PNB COMMUNICATIONS
}

"Brief communications" concerning vascular neurologic disorders in the same issue of Ann Neurol include the following:

Stroke-like episodes in autosomal recessive cytochrome oxidase deficiency. (Morin C, Dube J, Robinson BH et al. Ann Neurol March 1999;45:389392). Three patients with autosomal recessive Saguenay-Lac St-Jean cytochrome oxidase (COX) deficiency from Quebec, Canada, developed acute focal neurologic dysfunction and frontal hypodensities on CT, suggesting cerebral ischemia. Arteriography in 1 patient was normal during the acute episode. Some patients subsequently developed Leigh's disease (subacute necrotizing encephalomyelopathy). COX deficiency with congenital lactic acidosis is characterized by psychomotor retardation, hypotonia, and lactic acidemia.

Radiation-induced cerebral vasculopathy in children with neurofibromatosis and optic pathway glioma. (Grill J, Couanet D, Cappelli C et al. Ann Neurol March 1999;45:393-396). Neurofibromatosis 1 is a specific risk factor for radiation-induced cerebral vasculopathy. In a mean follow-up period of 7 years, 13 (19\%) of 69 children with NF-1 and optic pathway glioma (OPG) developed occlusive vasculopathy within 36 months of therapy. Radiation therapy should be avoided as first-line therapy for OPG when possible.

\section{INFECTIOUS DISORDERS}

The current issue of Arch Dis Child has three articles concerning neurologic abnormalities associated with infections:

Behavior and cognitive outcomes from middle ear disease. (Bennett KE, Haggard MP. Arch Dis Child 1999;80:28-35). Otitis media with effusion (OME) or "glue ear", the most common cause of hearing loss in children, is associated with an increased incidence of behavioral disorders (mainly neurotic and hyperactive behaviors, and clumsiness) at 5 years of age, and language and speech articulation problems at 10 years. Effects are modest but significant, emphasizing need for increased awareness of parents and preschool teachers, early referral, and perhaps more vigorous physician treatment of recurrent otitis media.

Long term neurological outcome of herpes encephalitis. (Lahat E, Barr J, Barkai G et al. Arch Dis Child 1999;80:69-71). Persistent neurological sequelae occurred in 10 of 28 children with HSE, followed for a mean of 5 years, and 2 died. A low Glasgow coma score is a risk factor for a poor outcome; a score over 10 predicts no neurologic sequelae and a good prognosis. Early diagnosis and treatment are emphasized. PCR is the accepted preferred diagnostic test.

Acute cerebellar ataxia with human parvovirus B19 infection. (Shimizu Y, Ueno T, Komatsu H et al. Arch Dis Child 1999;80:72-73). A first casereport of a 2 year-old boy with ACA and erythema infectiosum caused by PVB19. 